Matthias Forell, Gabriele Bellenberg \& Grit im Brahm

\title{
Das Gymnasium im Zuge fortschreitender Öffnung
}

\section{Zusammenfassung}

Der Beitrag beschreibt Öffnungsprozesse an Gymnasien in Nordrhein-Westfalen im Hinblick auf ihre Wettbewerbssituation und Profilierung. Grundlage bilden quantitative Daten zum Schulwesen des Ministeriums für Schule und Bildung des Landes NordrheinWestfalen (MSB NRW) sowie einzelschulische Daten der amtlichen Schulstatistik des Landesbetriebs Information und Technik Nordrhein-Westfalen (IT.NRW). Die Befunde $z u$ den Übergangsquoten, zur Durchlässigkeit und zur Verbleibwahrscheinlichkeit dieser neuen gymnasialen Schülerschaft werden vor dem Hintergrund der Typisierung von Schulen im Wettbewerb und Profilierungsprozess nach Maroy \& van Zanten (2009) analysiert.

Schlüsselwörter: Gymnasium, Öffnung, Profilierung, Wettbewerb, Durchlässigkeit

\section{The Gymnasium in the Course of Progressive Opening}

\section{Abstract \\ The article describes opening processes at Gymnasiums in North Rhine-Westphalia re- garding their competitive situation and profile raising. It is based on quantitative data on the school system from the Ministry of Schools and Education in North Rhine- Westphalia and individual school data from the official school statistics of IT.NRW. The findings on the transitional quotas, the permeability and the probability of remaining of this new student body are analyzed in view of the fact, which Maroy \& van Zanten (2009) describe as typing of schools in a competition and profiling process. Keywords: gymnasium, opening, profiling process, competition, permeability \\ 1. Die Bildungsexpansion und ihre Folgen für das Gymnasium in Nordrhein-Westfalen (NRW)}

Der zunehmende strukturelle Bedarf an Bildung erforderte einen groß angelegten Ausbau des deutschen Bildungssystems ab dem Ende der 1950er-Jahre. Durch die 
kontinuierliche Bildungsexpansion erhielt eine Vielzahl junger Menschen Zugang zu Realschulen und Gymnasien und damit die Möglichkeit, mittlere bzw. höhere Bildungsabschlüsse zu erwerben (Geißler, 2014, S. 334).

Im Rahmen der Bildungsexpansion wurden zudem vielfältige Möglichkeiten geschaffen, das Abitur auch an anderen Schulformen als dem Gymnasium zu erwerben. Dennoch stellt das Gymnasium nach wie vor die attraktivste Schulform in Deutschland dar. An keiner anderen Schulform ist die Nachfrage so hoch und konstant. Zudem ist das Gymnasium die einzige Schulform, die ausnahmslos in allen föderal organisierten Schulsystemen der Bundesländer angeboten wird. Für das Gymnasium lassen sich in der Folge der Bildungsexpansion Öffnungsprozesse beschreiben, die sich insbesondere beim Übergang von der Primar- in die Sekundarstufe manifestieren. Abbildung 1 veranschaulicht die erhebliche Ausweitung des Gymnasialbesuchs in NRW sowie die Entwicklung der Abiturquote derer, die als Eltern Schulformentscheidungen für ihre Kinder am Übergang nach der Grundschule treffen. Seit 2002 steigt die Übergangsquote zum Gymnasium wieder sukzessive an, nachdem sie zwischen 1988 und 1992 auf einem Niveau zwischen 36,4 und 36,8 Prozent stagnierte und sich danach bis 2001 auf 34,7 Prozent reduziert hatte. Im Schuljahr 2017/18 gingen in NRW 41 Prozent aller Schüler*innen in die fünfte Jahrgangsstufe des Gymnasiums über.

Abb. 1: Übergangsquoten in das Gymnasium in NRW und Bildungsstand in der Bundesrepublik Deutschland

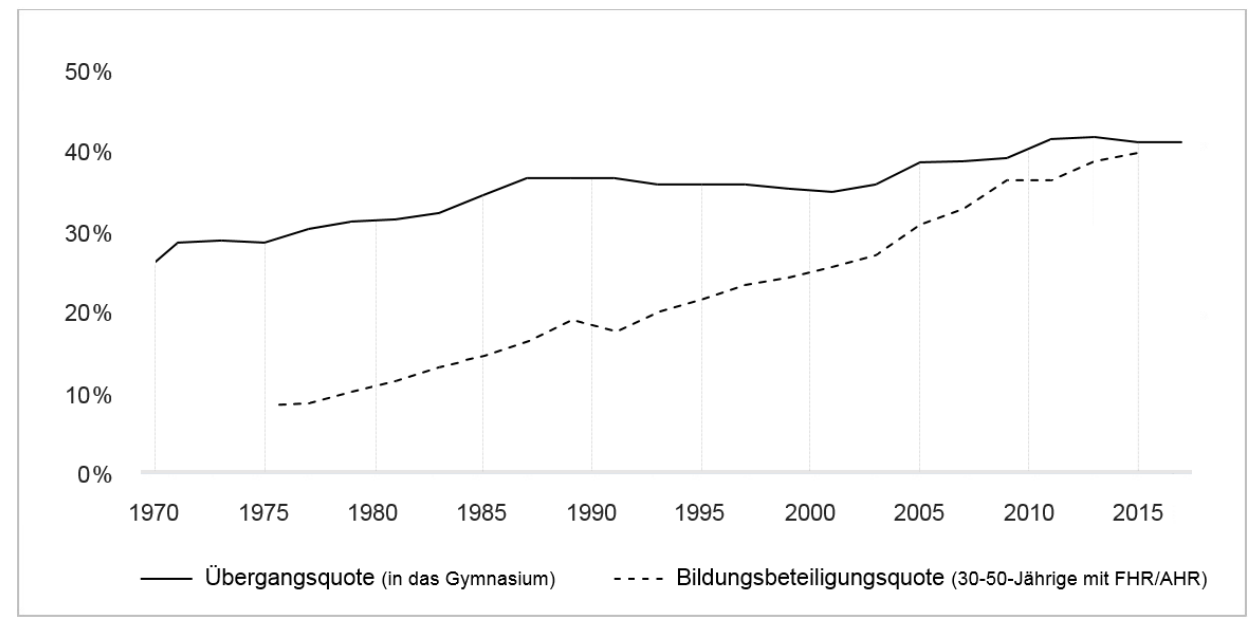

Quelle: Zusammenstellung auf Grundlage von MSB NRW, 2018, S. 240, und DESTATIS, $2018^{1}$

1 Dem Bildungsstand liegen abweichend zu allen anderen Daten Werte der Bundesrepublik Deutschland zu Grunde. Über den langen Beobachtungszeitraum wurde der Bildungsstand der 30- bis 50-Jährigen in NRW nur sehr lückenhaft erfasst, so dass die Bundesrepublik zum Vergleich herangezogen werden musste. 
Für die Dynamik der Übergangsquoten lassen sich als Erklärung die demographische Entwicklung (vgl. Abb. 2) sowie der Bildungsstand (Anteil mit Fachhochschulreife und allgemeiner Hochschulreife) der 30- bis 50-Jährigen (vgl. Abb. 1) heranziehen. Diese Altersgruppe schließt einen Großteil der Bevölkerung ein, die Kinder in dem Alter haben, in dem die Übergangsentscheidungen getroffen werden. Mit höheren Bildungsabschlüssen in der Elternschaft steigt zugleich auch der Wunsch der Eltern, ihren Kindern ebenfalls höhere Abschüsse zu ermöglichen (Rösner \& Stubbe, 2008, S. 305). Die Übergangsquoten nach der Grundschule steigen kongruent dazu an den beiden abiturführenden Schulformen Gymnasium und Gesamtschule. In den vergangenen vierzig Jahren hat sich deren Anteil in NRW auf 68,9 Prozent (GY: 41,0\%/GS: 27,9\%) im Schuljahr 2017/18 verdoppelt (MSB NRW, 2018, S. 240). Die Möglichkeit, auch an einer anderen Schule das Abitur erwerben $\mathrm{zu}$ können, ${ }^{2}$ führt $\mathrm{zu}$ einer Entlastung des Gymnasiums. Wurden 1978 knapp 95 Prozent der (Fach-)Abiture an Gymnasien erworben, sind es heute noch gut 75 Prozent (MSB NRW, 2018, S. 253 f.).

Die demographische Entwicklung (vgl. Abb. 2) offenbart, dass seit dem Jahr mit der geringsten Schüler*innenzahl beim Übertritt von der Grundschule in die Sekundarstufe I (150.161 im Jahr 1986) diese bis $2001 \mathrm{um}$ mehr als 50.000 (über 30 Prozentpunkte) angestiegen ist. Im gleichen Zeitraum stagniert die Übergangsquote in das Gymnasium bei rund 35 Prozent. Bis 1986 war es umgekehrt. Während die Schüler*innenzahl in ihrer Gesamtheit abnahm, stieg die Übergangsquote auf das Gymnasium kontinuierlich. Die Anzahl der Schüler*innen, die zwischen 1974 und 1986 vom Primar- in den Sekundarbereich übergangen sind, sank um über 85 Prozentpunkte, wohingegen die Übergangsquote zum Gymnasium um gut sieben Prozentpunkte anstieg. Diese gegenläufige Entwicklung von Demographie und gymnasialer Übergangsquote schreibt sich ab 2002 fort. Rösner (2013) bezeichnet in diesem Zusammenhang die „Demografie als intervenierende Variable“ (S. 183) und kommt zu dem Schluss: „Sinken die Schülerzahlen signifikant, steigen ebenfalls signifikant die Übergangsquoten in Gymnasien“ (ebd.).

Betrachtet man nun zudem die absoluten Schüler*innenzahlen des Gymnasiums in NRW, so lässt sich zum einen feststellen, dass das Jahr 1986 in zweierlei Hinsicht eine Zäsur darstellt: So gingen in diesem Jahr nicht nur die bisher wenigsten Kinder von der Grundschule in die weiterführenden Schulen über, sondern es markiert auch den Zeitpunkt, an dem das Gymnasium die Hauptschule als Schulform mit den meisten Schüler*innen in der Eingangsklasse des Sekundarbereiches abgelöst hat. Zum anderen zeigt die Betrachtung der absoluten Schülerinnenzahlen (vgl. Abb. 3), dass sich seit 1991 die Zahlen der Gymnasialschüler*innen in der fünften Jahrgangsstufe zwischen knapp 62.000 und gut 72.000 bewegen und dabei höchstens um etwa sechs Prozentpunkte pro Jahr variieren. Im gleichen Zeitraum schwank-

2 Ein (Fach-)Abitur an allgemeinbildenden Schulen kann in NRW sowohl an Gymnasien als auch an Gesamtschulen, Freien Waldorfschulen und Weiterbildungskollegs erworben werden. 
ten die Schüler*innenzahlen in der fünften Jahrgangsstufe der Realschulen von NRW zwischen rund 30.000 und 60.000, und die der Hauptschulen sanken von über 41.000 auf gut 5.000 .

Abb. 2: Demographische Entwicklung anhand der Schüler*innenzahlen am Übergang von der Grundschule in die Sekundarstufe I in NRW

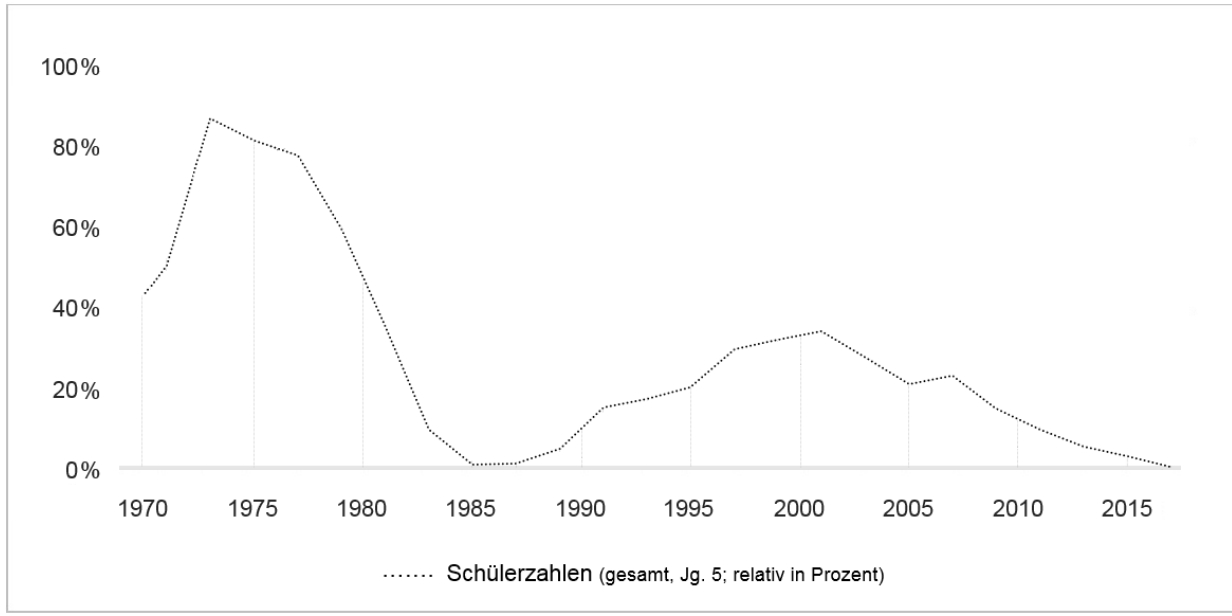

Quelle: eigene Berechnungen auf Datengrundlage von MSB NRW, 2018, S. $240^{3}$

Abb. 3: Schüler*innenzahlen in der fünften Jahrgangsstufe an den Gymnasien in NRW

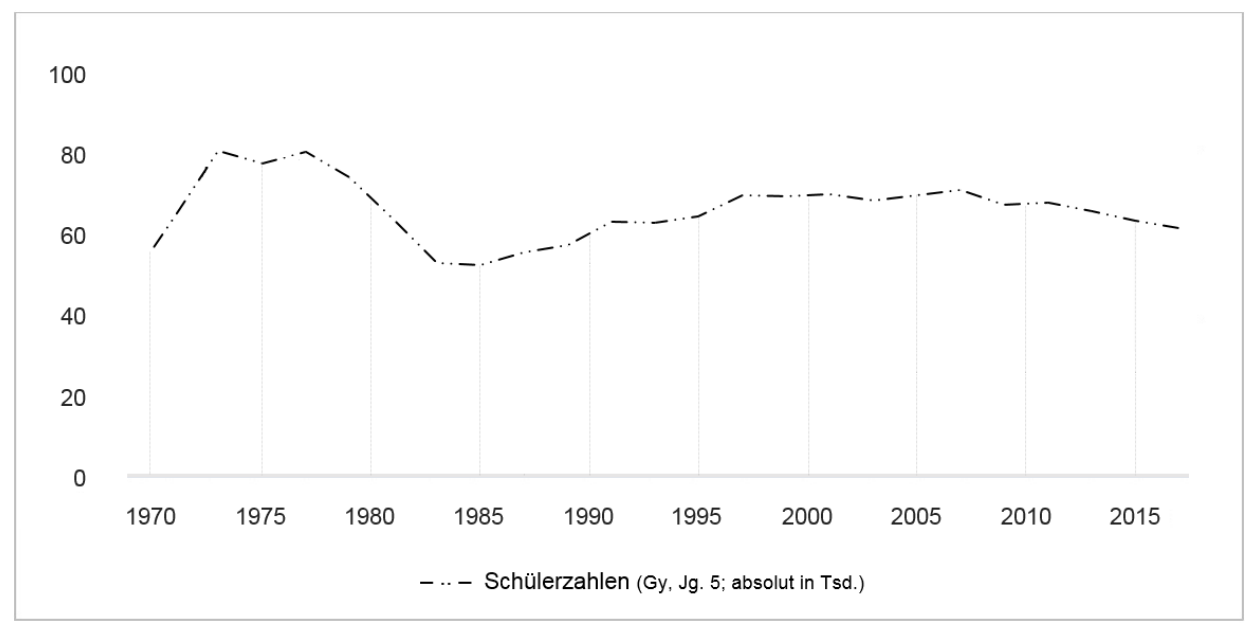

Quelle: eigene Zusammenstellung auf Grundlage von MSB NRW, 2018, S. 240

3 Die Werte wurden z-transformiert. Das Jahr mit der niedrigsten Schüler*innenzahl am Übergang in die Sekundarstufe I (1986: 150.161 Schüler*innen) wurde genullt. Die Schüle$\mathrm{r}^{*}$ innenzahlen der anderen Jahre wurden daran relativiert. 
Es kann festgehalten werden, dass es den Gymnasien gelingt, ihre Schüler*innenzahlen (und damit auch die Zahl der Schulen) relativ konstant zu halten. In Zeiten sinkender Schüler*innenzahlen steigen die Übergangsquoten in das Gymnasium, wohingegen sie bei weniger zur Verfügung stehenden Schüler*innen stagnieren oder leicht absinken. Die übrigen Schulformen müssen dagegen um eine einmal größere und einmal kleinere Schüler*innengruppe konkurrieren, deren Eltern zunehmend höhere Bildungsabschlüsse vorweisen können.

\section{Profilierung und Wettbewerb von Schulen in regionalen Bildungslandschaften}

Welche schulischen Angebote in einer Region zu finden sind, hängt einerseits von den schulstrukturellen Vorgaben des Landes ab und anderseits von den konkreten Entscheidungen des Schulträgers in Abstimmung mit angrenzenden Kommunen und sonstigen Akteuren. Die Nachfrage nach bestimmten Bildungsangeboten ist neben der Entwicklung der Schüler*innenzahl auch von elterlichen Wahlentscheidungen abhängig. Daher wird nun der Blick auf einzelschulische Wettbewerbskonstellationen und deren Marktlogiken innerhalb ihrer lokalen Schullandschaft gerichtet.

Existieren genügend Schulangebote in einer Bildungsregion oder steht gar ein regionales Überangebot zur Verfügung, befinden sich Schulen in einem Wettbewerb um Schüler*innen bzw. um die Entscheidungen der Eltern. Lokale und regionale sind dabei von globalen Wettbewerbskonstellationen $\mathrm{zu}$ unterscheiden. In mehrgliedrigen und größtenteils hierarchisch strukturierten Schulsystemen stehen die unterschiedlichen Schulformen nur bedingt miteinander in Konkurrenz, Schulen der gleichen Schulform innerhalb einer Bildungsregion hingegen schon (Zymek, 2009, S. 89). Der funktionellen Logik von Marktmechanismen liegen „Bewältigungshandlungen von Knappheitssituationen“ (Hermstein, 2018, S. 134) zugrunde. Somit wird bei gleichbleibendem gymnasialem Angebot der schulische Wettbewerb vor allem bei sinkenden Schüler*innenzahlen bedeutsam.

Dieser Wettbewerb gewinnt auch deshalb an Bedeutung, weil die Schulen seit den 1980er-Jahren Maßnahmen des New Public Managements unterworfen sind. Zu diesen gehört die Stärkung der Autonomie der Einzelschule (vor allem in Steuerungsfragen, die zuvor der Kultusadministration unterlagen) bei gleichzeitiger Erhöhung der Angebotsvielfalt und -flexibilität, die wiederum mehr Wahlmöglichkeiten eröffnet und die Nachfrage anregt. Ferner orientieren sich Qualitätssicherungsmaßnahmen nun weniger am Input als vielmehr am Output der Schulen bzw. an ihrem Outcome. Durch interschulische Vergleiche können in Folge dessen Hierarchien innerhalb der kommunalen Schullandschaft entstehen (u. a. Schweizer, 2012, S. 170; Zymek, 2009, S. 82; Baumert, Cortina \& Leschinsky, 2003, S. 136; Weiß, 2001, S. 69). 
Es haben sich dadurch Quasi-Märkte etabliert, die zwar durch die genannten Marktelemente bestimmt sind, jedoch in ihrer freien Entfaltung von der Kultusadministration eingeschränkt werden, da diese weiterhin u. a. die Aufsicht über die Schulen wahrnimmt und die Schulen ihr gegenüber rechenschaftspflichtig bleiben (Zymek, 2009, S. 82; Kussau \& Brüsemeister, 2007, S. 55; Weiß, 2001, S. 70).

Profilierungen ermöglichen es den Schulen, sich im Wettbewerb von anderen Schulen abzugrenzen und sich dadurch Wettbewerbsvorteile zu verschaffen. Macht man sich die weite Definition von Schulprofilen zu eigen, nach der unter einem Schulprofil „die Summe aller Merkmale [... zu verstehen ist], die die öffentliche Wahrnehmung einer Schule bestimmen [...]“ (Altrichter, Heinrich \& Soukup-Altrichter, 2011a, S. 11), gibt es neben curricularen Typisierungen erheblich differenziertere Profilierungsmerkmale. Ein Schulprofil ist als „die pädagogische Gestalt der Einzelschule“ (Clausen, Winkler \& Neu-Clausen, 2007, S. 747) zu verstehen, „um einen bewusst pädagogisch gestalteten Lern- und Lebensraum anzubieten“ (ebd.). Schulprofilierung beschreibt damit einen

„intentionalen Prozess, mit dem die Akteure einer Schule versuchen, das bestehende Profil dieser Schule - vor allem ihre unterrichtlichen und außerunterrichtlichen Angebote und ihre pädagogische Identität - weiterzuentwickeln“ (Altrichter et al., 2011a, S. 12).

Schulprofilierung setzt demzufolge also bewusste und steuernde Aktivitäten von Schulen voraus, soll aber auch „die regionalen Bedingungen berücksichtigen, unter denen der schulische Erziehungs- und Bildungsauftrag realisiert wird“ (Clausen et al., 2007, S. 747). Die Profilierung der Einzelschule findet also immer im Kontext der kommunalen Schullandschaft statt und steht in Wechselbeziehung mit den anderen (schulischen) Bildungsangeboten vor Ort. Maroy \& van Zanten (2009) bezeichnen diesen Umstand als „competitive interdependencies“ (S. 70), wonach jede Schule direkt von den Praktiken derjenigen Schulen betroffen ist, die im gleichen Bezirk oder in der Nachbarschaft liegen. Diese Abhängigkeiten beziehen sich in erster Linie auf die Schüler*innen, um die die Schulen konkurrieren, aber auch auf Ressourcen wie z.B. Lehrkräfte oder Räumlichkeiten und finanzielle Zuwendungen. Im oben skizzierten Quasi-Markt kommunaler Schullandschaften lässt sich mit dem Fokus auf die Schülerschaft konstatieren, dass der Wettbewerbsdruck Segregation zwischen den Schulen insofern fördert, als diese nicht alleine um die Schüler*innen allgemein konkurrieren, sondern vor allem um solche mit bestimmten Fähigkeiten, d.h. um lernbereite und leistungsstarke, deren erfolgreiche Anwerbung wiederum zu distinguierten Schulprofilen führt (Maroy \& van Zanten, 2009, S. 71-73; Zymek, 2009, S. 83; Weiß, 2001, S. 77).

In diesem Zusammenhang unterscheidet Zymek (2009) konkurrenzlose Solitärschulen, die sich relativ unberührt von Wettbewerbsüberlegungen entwickeln kön- 
nen, von sogenannten Sammelschulen. Diese verortet er vor allem in Gymnasien im Kernbereich von Großstädten, die versuchen,

„in der historisch neuen Konkurrenzsituation durch besondere curriculare Profile (z. B. Latein ab der fünften Jahrgangsstufe, bilinguale Klassen, Musikklassen) weiterhin eine Schülerschaft aus dem ganzen Stadtgebiet und dem Umland anzuziehen" (S. 90 f.).

Er entwirft damit das Schulprofil als eine Bewährungsstrategie in einer spezifischen lokalen Konkurrenzsituation, die durch Vernetzung in einem bestimmten Sozialmilieu entstanden ist und dieses widerspiegelt.

In einer internationalen Studie konnten Maroy \& van Zanten (2009) zeigen, dass Profilbildungsprozesse $\mathrm{zu}$ einer Hierarchisierung zwischen Schulen sowie zu einer Segregation ihrer Schülerpopulation führen können. Die spezifischen Handlungsweisen der Schulen werden dabei zwar durch nationale institutionelle Kontexte vorstrukturiert, aber durch lokale Faktoren überformt. Die Besonderheit des regionalen Kontextes beschreiben die Autoren durch Wettbewerbsvariablen, denen die Schulen ausgesetzt sind (s.o. „competitive interdependencies“) und den Status, den die Schulen innerhalb der lokalen Hierarchie innehaben („position in hierarchy“; ebd., S. 72). Für beide Variablen lassen sich jeweils zwei Ausprägungen klar voneinander abgrenzen. Die Wettbewerbssituation kann als offen und instabil beschrieben werden. Demgegenüber kann sie aber auch begrenzt und stabil sein. Für den Status der jeweiligen Einzelschule lassen sich Schulen mit einer hohen Position von denen unterscheiden, die sich auf einem mittleren oder niedrigen Rang befinden. Maroy \& van Zanten (ebd., S. 73-75) arbeiten auf dieser Grundlage vier verschiedene idealtypische schulische Handlungslogiken heraus (vgl. Tab. 1):

Tab. 1: Schulische Handlungslogiken im Kontext von Wettbewerb und Profilierung

\begin{tabular}{l|l|l}
\hline $\begin{array}{c}\text { Wettbewerb } \\
\text { Status/Position }\end{array}$ & \multicolumn{1}{c}{ offen } \\
\hline $\begin{array}{l}\text { hoher Status/ } \\
\text { hoch positioniert }\end{array}$ & $\begin{array}{l}\text { LOGIK DER EROBERUNG } \\
\text { Schulen offerieren spezifische } \\
\text { curriculare Angebote. Sie können } \\
\text { Schülerinnen und Schüler auswählen } \\
\text { und selektiv aufnehmen. }\end{array}$ & $\begin{array}{l}\text { begrenzt } \\
\text { STATUS QUO } \\
\text { kufrechterhaltung der Reputation, } \\
\text { des Anvestition in die Innovation }\end{array}$ \\
\hline $\begin{array}{l}\text { niedriger Status/ } \\
\text { niedrig positioniert }\end{array}$ & $\begin{array}{l}\text { LOGIK DER DIVERSIFIZIERUNG } \\
\text { Diversifikation des Bildungsangebots, } \\
\text { Vermeidung der Benachteiligung der } \\
\text { Schule }\end{array}$ & $\begin{array}{l}\text { LOGIK DER ADAPTION } \\
\text { unfreiwillige Etablierung in einer } \\
\text { Nische }\end{array}$ \\
\hline
\end{tabular}

Quelle: eigene Darstellung nach Maroy \& van Zanten, 2009 
Schulprofile werden besonders bei Übergängen virulent, wenn Eltern Wahloptionen für bestimmte Einzelschulen haben und keine Bindung an eine Schulformempfehlung besteht. In solchen Situationen versuchen Schulen, im Wettbewerb um eine größtmögliche Anzahl bestmöglicher Schüler*innen erfolgreich zu sein. Deshalb können sowohl die Schüler*innenzahlen der fünften Jahrgangsstufe (Kap. 3) als auch die Schulformempfehlungen der Schüler ${ }^{\star}$ innen (Kap. 4) als zentrale Erfolgsindikatoren für einen schulischen Wettbewerb angesehen werden. Der Erfolg einer Schule in dieser quantitativen „first-order competition“ ist Voraussetzung für die Möglichkeit, bei der qualitativen „second-order competition“ mitwirken zu können (ebd., S. 71).

Erfolg im Wettbewerb liegt vor, wenn nicht nur eine hohe Zahl von Schüler*innen rekrutiert werden kann, sondern zugleich viele leistungsstarke bzw. solche mit einem hohen sozialen Status. Mit diesem Wettbewerb zweiter Ordnung sinkt die Sensibilität gegenüber den Problemen sozialer Auslese (Altrichter, Heinrich \& Soukup-Altrichter, 2011b, S. 226). So kann es innerhalb einer regionalen Schullandschaft zu einer Hierarchisierung von Schulprofilen kommen, mit der Folge, dass bestimmte Schüler*innengruppen von attraktiven Angeboten ausgeschlossen werden, während für Schüler*innen mit belasteten Schulkarrieren nur Schulen oder Jahrgangsstufen mit ungünstigen Lernmilieus zur Verfügung stehen (ebd., S. 231). Gegenstand des Beitrages ist dabei nicht die soziale Selektivität auf Individualebene, die durch eine derartige Bildungsbenachteiligung hervorgerufen wird, sondern die Öffnung von Gymnasien im Kontext von Wettbewerb und Profilierung im lokalen Raum.

\section{Entwicklung der Schüler*innenzahlen in der fünften Jahrgangsstufe}

In den Zeitraum des letzten demographisch bedingten Rückgangs der Schüler*innenzahlen seit 2001 fallen zentrale bildungspolitische Entscheidungen des Ministeriums für Schule und Bildung des Landes Nordrhein-Westfalen (MSB NRW), die maßgeblichen Einfluss auf den Übergang zum Gymnasium haben. So wurde zunächst zum Schuljahr 2005/06 flächendeckend vom neunjährigen (G9) auf einen achtjährigen (G8) gymnasialen Bildungsgang umgestellt. Im Schuljahr darauf wurden die Schulformempfehlungen bindend. Eltern, deren Kinder nicht für das Gymnasium empfohlen wurden, konnten es nun nicht mehr anwählen. Eine Abweichung von einer nichtgymnasialen Schulformempfehlung war nur noch nach besonderen Hürden (u.a. durch die erfolgreiche Teilnahme am Prognoseunterricht) möglich. Ab dem Schuljahr 2011/12 wurde die Bindung an die Grundschulempfehlung wieder aufgehoben. Eltern haben nun wieder die Möglichkeit, sich unabhängig von der Schulformempfehlung ihrer Kinder für eine Schulform und Schule ihrer Wahl zu entscheiden. Im gleichen Schuljahr wurde ein Schulversuch eingeführt, der zwölf Gymnasien wieder die Möglichkeit gegeben hat, von einem acht- auf einen neuen neunjährigen gymnasialen Bildungsgang $\left(\mathrm{G} 9_{\text {neu }}\right.$ ) umzustellen. 
Im Wettbewerb um (gute bzw. die besten) Schüler*innen haben diese Modellgymnasien in der Sekundarstufe I ein Schuljahr mehr zur Verfügung und können diese zusätzliche Zeit zur Profilierung sowie als Alleinstellungsmerkmal innerhalb ihrer kommunalen Schullandschaft nutzen. Zudem ermöglicht ihnen die gleichzeitige Aufhebung der Bindung der Schulformempfehlung, wieder um alle Schüler*innen, die die Grundschule zu einer weiterführenden Schule verlassen, zu werben. Auch vor dem Hintergrund der großflächigen Rückkehr von NRW zum neunjährigen gymnasialen Bildungsgang ab dem Schuljahr 2019/20 verdienen die Modellgymnasien des Schulversuchs zu G9 ${ }_{\text {neu }}$ im Folgenden für die Öffnung der Gymnasien in NRW besondere Beachtung.

Der Systematik schulischer Handlungslogiken nach Maroy \& van Zanten (2009; vgl. Tab. 1) folgend, zeigt sich unter den Modellgymnasien ein differenziertes Bild. Exemplarisch sollen hier die Schulen der beiden Schultypen näher betrachtet werden, die sich größtmöglich voneinander abgrenzen:

1) Solitärschulen in begrenztem Wettbewerb, die einen hohen Status aufweisen (Logik des Profits vom Status Quo): Diese Schulen konzentrieren sich auf die Aufrechterhaltung ihrer Reputation und ihrer etablierten Selektionspraktiken.

2) Sammelschulen in offenem Wettbewerb, die über einen mittleren oder niedrigen Status verfügen (Logik der Diversifizierung): Diese Schulen differenzieren ihr schulisches Angebot größtmöglich aus und fördern die Vielfalt in der Zusammensetzung ihrer Schülerschaft.

Bei den beiden im Folgenden betrachteten Solitärschulen handelt es sich um zwei Gymnasien, die nicht nur wegen ihrer Lage über eine Monopolstellung in ihrer Region verfügen, da sie keine Konkurrenzschulen im direkten Umfeld haben, sondern auch aufgrund ihres spezifischen pädagogischen Konzepts über eine hohe Reputation verfügen und sich beide in freier Trägerschaft befinden. Dem gegenüber stehen vier Gymnasien, die durch ihre Lage in einem Stadtgebiet einer offenen Wettbewerbssituation ausgesetzt sind. Diese Sammelschulen stehen in einem starken Konkurrenzverhältnis zu anderen Schulen in einem Umkreis von etwa fünf Kilometern. In ihrer Großstadt nehmen sie in der Hierarchie der Gymnasien eher eine niedrige Position ein, was sich teilweise auch über ihre (sozio-)geographische Lage erklären lässt. Dadurch konkurrieren sie nicht nur mit anderen Gymnasien um (gute) Schülerinnen, sondern auch mit Gesamtschulen und Realschulen.

Entsprechend der beiden oben genannten schulpolitischen Maßnahmen zum Schuljahr 2011/12 (Aufhebung der Bindung der Schulformempfehlung sowie Start des Schulversuchs $\mathrm{zu}$ G9 ${ }_{\text {neu }}$ ) wird für die Analyse der Schüler*innenzahlentwicklung das vorangegangene Schuljahr 2010/11 als Ausgangsjahr zugrunde gelegt. Dabei zeigt sich, dass - trotz des Rückgangs der in den Sekundarbereich übergehenden Schüler*innen um rund 14 Prozentpunkte zwischen 2006 und 2014 - sowohl 
die Solitär- als auch die Sammelschulen ihre Schüler*innenzahlen in der fünften Jahrgangsstufe steigern konnten. Gleichwohl offenbaren sich deutliche Unterschiede zwischen den beiden Schultypen. Während es den beiden Solitärschulen gelungen ist, die Zahl ihrer Fünftklässler*innen sukzessive zu steigern, konnten die vier Sammelschulen, die im Schuljahr 2010/11 noch von Schulschließungen bedroht waren, die Zahl ihrer Fünftklässler*innen drastisch erhöhen und den Wettbewerb in ihrer kommunalen Schullandschaft um Schüler*innen mit Abituraspiration durch ihr profilbildendes Alleinstellungsmerkmal G9 ${ }_{\text {neu }}$ für sich entscheiden.

Es bleibt festzuhalten, dass an den Gymnasien beider Schultypen 2016 rund 30 Prozent mehr Schüler*innen die fünfte Jahrgangsstufe besuchen, als dies noch zehn Jahre zuvor der Fall gewesen ist. Bei durchschnittlich 90 Schüler*innen, die 2006 auf jedes der sechs Modellgymnasien übergegangen sind, ist dies gleichbedeutend mit der Einrichtung einer zusätzlichen Eingangsklasse.

\section{Zusammensetzung der Schülerschaft in der fünften Jahrgangsstufe}

Die Frage, ob die Öffnung der Gymnasien auch mit einer veränderten Schülerklientel einhergeht, bedarf zunächst einer Nachzeichnung der in dem betrachteten Zeitraum vergebenen Schulformempfehlungen durch die Grundschullehrkräfte (Abb.4). Aus dieser geht hervor, dass sich die Schulformempfehlungen der abgebenden Grundschulen in NRW zwischen den Schuljahren 2009/10 und 2016/17 nur marginal verändert haben. Dies trifft ebenso auf die im Spezielleren untersuchten Modellregionen zu. $^{4}$

Verließen 2009 etwas über 35 Prozent der Schülerinnen landesweit ihre Grundschulen mit einer uneingeschränkten Gymnasialempfehlung, so waren es 2016 mit 34,2 Prozent ein Prozentpunkt weniger. Dies war der niedrigste Anteil uneingeschränkter Gymnasialempfehlungen zwischen 2009 und 2016; 2012 war dieser mit 36,6 Prozent am höchsten. Etwas größer fällt der Unterschied zwischen den Schüler*innengruppen aus, die keine Gymnasialempfehlung erhalten haben (zwischen dem Höchstwert von 55,9\% im Jahre 2009 und dem niedrigsten mit 52,7\% im Jahre 2015). Die Verluste beider Empfehlungsgruppen erhöhen den Anteil der eingeschränkt Gymnasial-Empfohlenen, deren Anteil in diesem Zeitraum sukzessive um 3,1 Prozentpunkte von 8,9 auf nun 12,0 Prozent anstiegen ist (MSB NRW, 2018, S. 42).

Die Erhöhung des Anteils von Schüler*innen mit einer eingeschränkten Gymnasialempfehlung spiegelt sich in der Zusammensetzung der gymnasialen Schülerschaft in der fünften Jahrgangsstufe wider. In Abbildung 5 lassen sich der schrittwei-

4 Das MSB NRW erfasst die Schulformempfehlungen erst seit dem Schuljahr 2009/10. 
Abb. 4: Zusammensetzung der Schülerschaft in der fünften Jahrgangsstufe nach Schulformempfehlung am Gymnasium in NRW (ausschließlich keine oder eingeschränkte Gymnasialempfehlung)

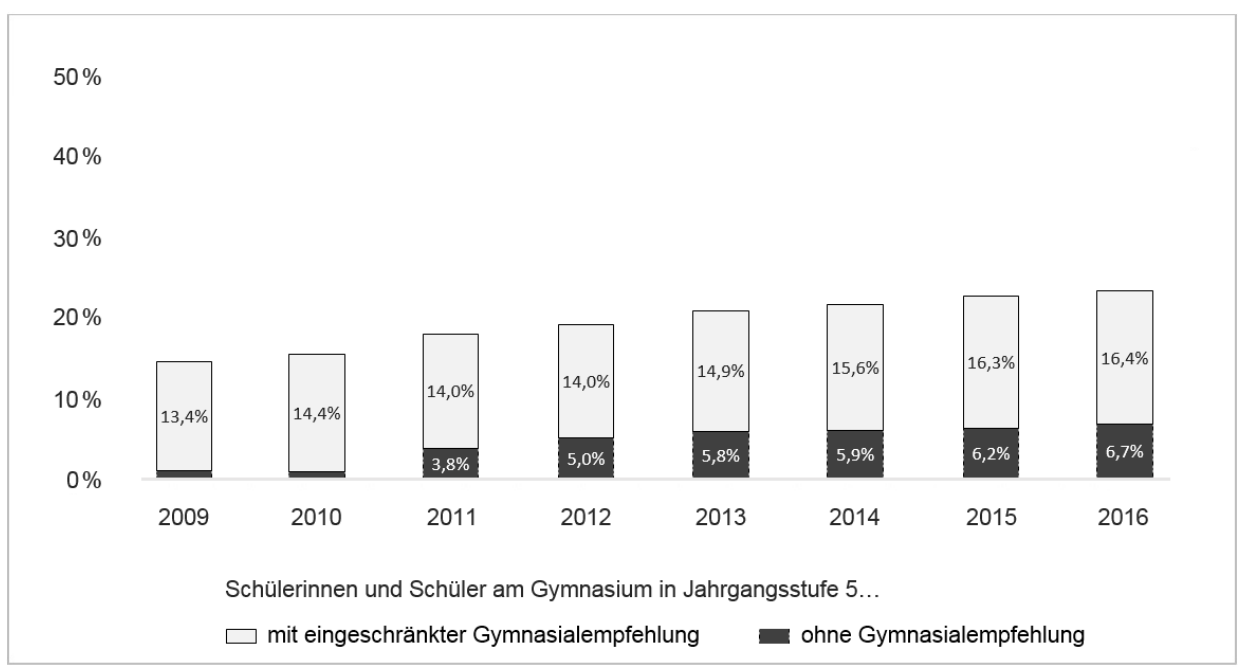

Quelle: Zusammenstellung auf Datengrundlage von MSB NWR, 2010-2017, S. 38-44

se Anstieg der eingeschränkt für das Gymnasium Empfohlenen und deren Übergangspraxis nachverfolgen. Demnach wechselten diese Schüler*innen proportional zu ihrem Anstieg an der Gesamtschülerschaft in gleichem Maße von der Grundschule zum Gymnasium. Ebenso schrittweise erfolgte der Anstieg an Schüler*innen, die über keine Gymnasialempfehlung verfügen. In den Jahren mit bindender Schulformempfehlung machte der Anteil nicht für das Gymnasium empfohlener Schüler*innen nur etwa ein Prozent der gymnasialen Schülerschaft in der fünften Jahrgangsstufe aus. Mit dem Wegfall der Bindung an die Empfehlung der Grundschullehrkräfte zum Schuljahr 2011/12 änderte sich das abrupt. In den vergangenen sechs Schuljahren stieg der Anteil Nicht-Gymnasial-Empfohlener von 3,8 auf nunmehr 6,7 Prozent. Im Schuljahr 2009/10 verfügten noch mehr als 85 Prozent der Gymnasiast*innen in der fünften Jahrgangsstufe über eine uneingeschränkte Gymnasialempfehlung; im Schuljahr 2016/17 sank dieser Anteil auf etwas weniger als 77 Prozent.

Diese eindeutige Tendenz zur Öffnung der nordrhein-westfälischen Gymnasien für eine neue Schülerschaft lässt sich allerdings nicht mehr über den leichten Rückgang der von den Grundschullehrkräften ausgesprochenen uneingeschränkten Gymnasialempfehlungen erklären. Vielmehr machen Eltern zunehmend von der Möglichkeit Gebrauch, von der Schulformempfehlung abzuweichen und ihre Kinder trotz fehlender Empfehlung an einem Gymnasium anzumelden. Dabei stellt sich die Frage, warum die Gymnasien diese Schüler*innen aufnehmen und noch dazu in einem so hohen Maße? 


\subsection{Zusammensetzung der Schülerschaft an den Solitär- und Sammelschulen}

In Zeiten abnehmender Schüler*innenzahlen erhöht sich - sofern die Gymnasien darum bestrebt sind, ihre Schüler ${ }^{\star}$ innenzahlen wenigstens auf einem konstanten Niveau zu halten - der Konkurrenzdruck zwischen den Gymnasien und den anderen Schulen innerhalb der kommunalen Schullandschaft. Insbesondere Schulen in offenem Wettbewerb, die sich in ihrer lokalen Hierarchie eher in einer niedrigen Position befinden, sind dann unter Umständen dazu gezwungen, weniger gute Schüler*innen aufzunehmen.

Wie oben beschrieben, verfügen die vier am Schulversuch teilnehmenden Sammelschulen über einen mittleren bis niedrigen Status in ihrem Umfeld. Daraus ergibt sich, dass sie in der Regel im Wettbewerb um (gute) Schüler*innen anderen Gymnasien mit einem höheren Status unterlegen sind. Mit der Teilnahme am Schulversuch zu G9 $9_{\text {neu }}$ konnten sie ihre Schüler*innenzahlen beim Übergang in die Sekundarstufe I erheblich steigern. Dies geht mit einer verstärkten Öffnung einher, die einen Anstieg der Schülerschaft ohne uneingeschränkte Gymnasialempfehlung zur Folge hat. Dabei gilt: Je höher die Konkurrenz zu anderen Gymnasien, desto stärker schreitet diese Öffnung für eine neue Schülerklientel voran.

Abb. 5: Zusammensetzung der Schülerschaft in der fünften Jahrgangsstufe nach Schulformempfehlung an den Modellgymnasien nach ihrer Wettbewerbssituation und Trägerschaft (ausschließlich keine oder eingeschränkte Gymnasialempfehlung)

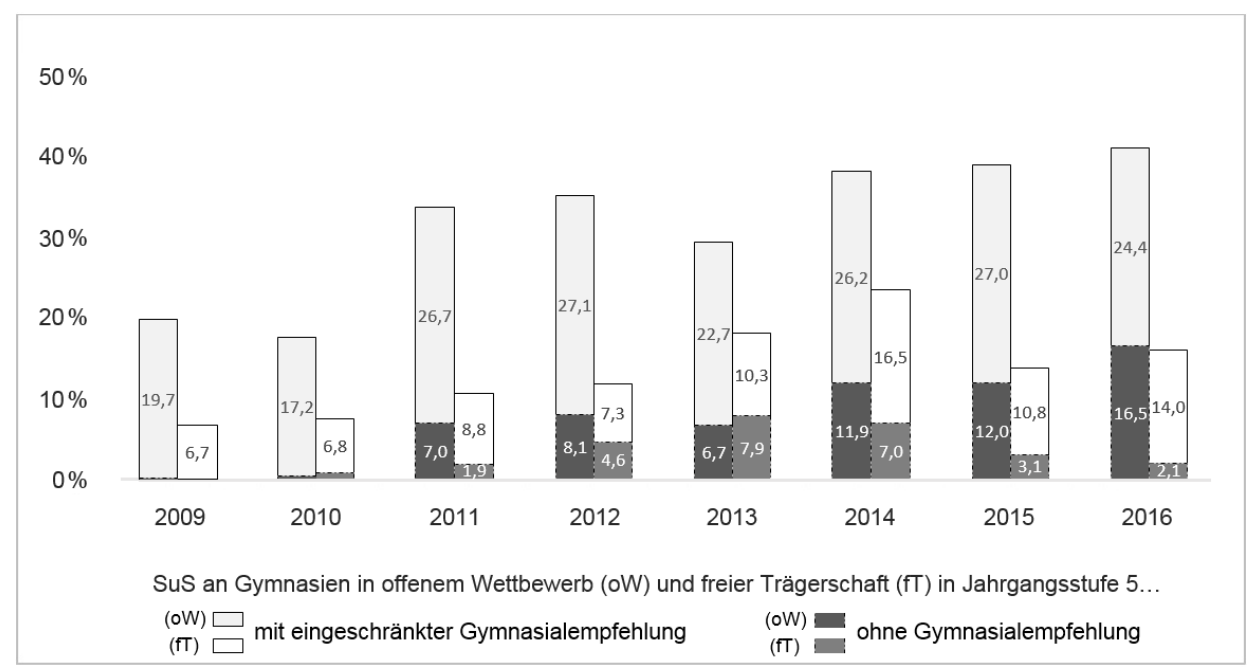

Quelle: eigene Berechnungen auf Datengrundlage von IT.NRW 
Abbildung 5 verdeutlicht die Unterschiede zwischen den beiden Schultypen. Von allen Modellgymnasien sind es die zwei in freier Trägerschaft, die auch im Vergleich zur fortschreitenden Öffnung im Mittel aller Gymnasien Nordrhein Westfalens (vgl. Abb. 4) den niedrigsten Gesamtanteil an Schüler*innen ohne uneingeschränkte Gymnasialempfehlung in ihren fünften Jahrgangsstufen aufweisen.

Schon in den beiden Jahren vor Beginn des Schulversuchs und der Aufhebung der bindenden Schulformempfehlung lässt sich die durch den hohen Status bedingte selektive Aufnahmepraxis der Gymnasien in freier Trägerschaft nachvollziehen. Im Vergleich zu den Schulen in offenem Wettbewerb zeigt sich, dass diese zwei Gymnasien in diesem Zeitraum nur halb so viele Schüler*innen ohne uneingeschränkte Gymnasialempfehlung aufnahmen. Im Schuljahr 2016/17 hatten an den Gymnasien in offenem Wettbewerb mehr als 40 Prozent der Fünftklässler*innen keine uneingeschränkte Gymnasialempfehlung. Sechs bzw. sieben Jahre zuvor lag dieser Wert noch bei weniger als 20 Prozent. Nachdem der Anteil derjenigen Schüler*innen ohne Gymnasialempfehlung von 2010 bis 2011 von unter einem Prozent auf sieben Prozent angestiegen ist, hat er sich seitdem noch einmal um über neun Prozent auf 16,5 Prozent erhöht. An den Gymnasien in freier Trägerschaft sind es im gleichen Jahr nur 2,1 Prozent.

Stellt man nun einen Zusammenhang zwischen den Befunden zur Schüler*innenzahlentwicklung (Kap. 3) und Abbildung 5 her, legt dieser nahe, dass der quantitative Erfolg im Wettbewerb um (gute) Schüler*innen mit einer Schülerschaft einhergeht, die qualitativ (nach ihrer Schulformempfehlung) weniger geeignet für das Gymnasium scheint. Ob damit tatsächlich eine erhöhte Selektion verbunden ist und sich dies auf eine fehlende Passung zwischen einer bestimmten Schüler*innengruppe und den Anforderungen des Gymnasiums zurückführen lässt, wird im Folgenden auf Basis der Analysen zur Durchlässigkeit und Verbleibwahrscheinlichkeit erörtert.

\subsection{Durchlässigkeit innerhalb der Sekundarstufe I}

Nach den positiven Effekten der Öffnung des Gymnasiums auf die „Übergangsauslese" (Tillmann, 2008, S. 54) des gegliederten Schulsystems zeigt sich mit Blick auf die horizontale Durchlässigkeit, wie selektiv sich das sich öffnende Gymnasium im Verlauf der Sekundarstufe I darstellt. Für die Analyse werden die Selektionsinstrumente Abschulung und (unfreiwillige) Klassenwiederholung herangezogen. Unter Abschulung wird hier der Abgang in eine andere Schulform verstanden. Wechsel an andere Gymnasien werden dabei nicht berücksichtigt. Die dadurch ermittelten Selektionsquoten unterscheiden sich wesentlich von den Durchgangsquoten, die durch Schülerinnenzahlveränderungen beim Wechsel in nächsthöhere Jahrgangsstufen die Erreichbarkeitswahrscheinlichkeit ermitteln. In diesem Kontext kann nur der Saldo aus den $\mathrm{Zu}$ - und Abgängen an das bzw. vom Gymnasium errechnet werden 
(Rösner, 2013, S. 185), ohne jedoch differenzierte Aussagen über die „Verluste“ und „Gewinne“ innerhalb der Schülerschaft treffen zu können.

Die erhöhten Schüler*innenzahlen der Modellgymnasien führen je nach Wettbewerbssituation zu unterschiedlichen Auslesestrategien. Dabei gelingt es den Solitärschulen in freier Trägerschaft am besten, ihre Schülerschaft bis zum Ende der Mittelstufe zu halten. Die Modellgymnasien in offenem Wettbewerb schulen dagegen vergleichsweise häufiger $a b$ und setzen auch das Instrument der Klassenwiederholungen häufiger ein (vgl. Tab. 2). Die beiden Gymnasien in freier Trägerschaft haben in jeder der vier analysierten Kohorten bis zum Beginn der neunten Jahrgangsstufe sowohl die wenigsten Abschulungen als auch die wenigsten Klassenwiederholungen aufzuweisen. Insgesamt verlassen immer weniger als zehn Prozent der Schüler*innen ihren Ursprungsjahrgang aus der fünften Jahrgangsstufe, wohingegen die vier untersuchten Sammelschulen in offenem Wettbewerb eine Selektionsquote zwischen 15 und 20 Prozent aufweisen. Die einzige Ausnahme bildet mit 12,6 Prozent die Schüler*innengruppe, die im Schuljahr 2012/13 den fünften Jahrgang besuchte.

Tab. 2: Selektionsquoten bis zum Beginn der neunten Jahrgangsstufe im Schultypenvergleich (kumuliert)

\begin{tabular}{|c|c|c|c|c|c|c|c|c|c|}
\hline & & \multicolumn{8}{|c|}{ Schüler*innen in Jahrgang 5 im Schuljahr } \\
\hline & & \multicolumn{2}{|c|}{$2009 / 10$} & \multicolumn{2}{|c|}{ 2010/11 } & \multicolumn{2}{|c|}{$2011 / 12$} & \multicolumn{2}{|c|}{$2012 / 13$} \\
\hline & & Jg. 7 & Jg. 9 & Jg. 7 & Jg. 9 & Jg. 7 & Jg. 9 & Jg. 7 & Jg. 9 \\
\hline freie & Abgänge & $2,5 \%$ & $5,6 \%$ & $2,2 \%$ & $6,3 \%$ & $2,8 \%$ & $4,6 \%$ & $2,7 \%$ & $5,0 \%$ \\
\hline \multirow{2}{*}{$\begin{array}{l}\text { Träger- } \\
\text { schaft }\end{array}$} & $\mathrm{KW}$ & $1,5 \%$ & $2,0 \%$ & $0.9 \%$ & $1,3 \%$ & $1,4 \%$ & $2,3 \%$ & $3,2 \%$ & $4,1 \%$ \\
\hline & gesamt & $4,0 \%$ & $7,6 \%$ & $3,1 \%$ & $7,6 \%$ & $4,2 \%$ & $6,9 \%$ & $5,9 \%$ & $9,1 \%$ \\
\hline \multirow{3}{*}{$\begin{array}{l}\text { Wett- } \\
\text { bewerb } \\
\text { offen }\end{array}$} & Abgänge & $3,6 \%$ & $5,5 \%$ & $0,9 \%$ & $11,1 \%$ & $5,8 \%$ & $11,0 \%$ & $7,5 \%$ & $8,6 \%$ \\
\hline & $\mathrm{KW}$ & $2,9 \%$ & $10,0 \%$ & $0,9 \%$ & $8,3 \%$ & $2,9 \%$ & $7,3 \%$ & $1,4 \%$ & $4,0 \%$ \\
\hline & gesamt & $6,5 \%$ & $15,5 \%$ & $1,8 \%$ & $19,4 \%$ & $8,7 \%$ & $18,3 \%$ & $8,9 \%$ & $12,6 \%$ \\
\hline
\end{tabular}

Quelle: eigene Berechnungen auf Datengrundlage von IT.NRW

Grundsätzlich müssen mehr Schüler*innen ein Gymnasium verlassen als aus einer anderen Schulform aufsteigen. In NRW kamen im Verlauf der Sekundarstufe I im Schuljahr 2010/11 auf einen Aufstieg sieben Abstiege (Bellenberg, 2012, S. 147). Im Vergleich dazu weisen die Modellgymnasien in freier Trägerschaft in den vier untersuchten Kohorten ein Verhältnis von Auf- zu Abstiegen von 1:4 bis zum Beginn der neunten Jahrgangsstufe auf. Bei den Modellgymnasien in offenem Wettbewerb variieren die Verhältnisse zwischen 1:6 und 1:12. 
Darüber hinaus fällt auf, dass im Durchschnitt die Bereitschaft größer ist, Teile der Schülerschaft abzugeben, wenn sich mehr Schüler*innen einer Jahrgangsstufe in einer Kohorte befanden. Auf diesen Zusammenhang wiesen Mauthe \& Rösner bereits 1998 (S. 116-119) in ihrer Erhebung zur Durchlässigkeit für NRW und BadenWürttemberg von 1980 bis 1996 hin. Diese Dynamik kann zunächst unabhängig von der Zusammensetzung der Schülerschaft beobachtet werden.

\subsection{Verbleibwahrscheinlichkeit von Schülerinnen nach ihrer Schulformempfehlung}

Wenn erhöhte Schüler*innenzahlen zu höheren Abschulungsquoten führen, liegt die Vermutung nahe, dass dies auch mit einer vermehrten Abschulung von nicht gymnasial-empfohlenen Schüler*innen einhergeht. Allerdings müssen an den Modellgymnasien in freier Trägerschaft anteilig weit mehr Schüler*innen mit eingeschränkter Gymnasialempfehlung in eine andere Schulform wechseln als Schüler*innen, die über eine reine Real- oder Hauptschulempfehlung verfügen. Erwartungskonform haben die Schülergruppen ohne Gymnasialempfehlung der Modellgymnasien in offenem Wettbewerb sehr viel geringere Verbleibwahrscheinlichkeiten als ihre Mitschüler*innen mit einer eingeschränkten Gymnasialempfehlung. Tabelle 3 schlüsselt differenziert nach Schultyp und Schulformempfehlung die Verbleibwahrscheinlichkeiten der jeweiligen Schüler*innengruppen bis nach der Erprobungsstufe auf und offenbart, dass die geäußerte Vermutung in ihrer generellen Aussage nicht haltbar ist. So verbleibt in beiden Schultypen zwar der größte Anteil derer, die über eine uneingeschränkte Gymnasialempfehlung verfügen (mit Ausnahme eines Schuljahres immer über 95 Prozent). Im Schuljahr 2013/14 - dem Schuljahr mit den mit Abstand höchsten Schüler*innenzahlen - verlassen bis zur siebten Jahrgangsstufe sogar mehr als die Hälfte der Schüler*innen ohne Empfehlung eines dieser Modellgymnasien. Im Umkehrschluss heißt das aber auch, dass der überwiegende Teil der nicht uneingeschränkt gymnasialempfohlenen Schüler*innen auch nach der Erprobungsstufe noch an den Modellgymnasien verbleibt. 
Tab. 3: Verbleibwahrscheinlichkeit der Schülergruppen nach ihrer jeweiligen Schulformempfehlung an den Modellgymnasien bis zum Beginn der siebten Jahrgangsstufe

\begin{tabular}{|c|c|c|c|c|c|c|c|}
\hline & \multirow{3}{*}{$\begin{array}{l}\text { Gymnasial- } \\
\text { empfehlung }\end{array}$} & \multicolumn{6}{|c|}{ Jahrgangsstufe 5 im Schuljahr } \\
\hline & & $2009 / 10$ & $2010 / 11$ & $2011 / 12$ & $2012 / 13$ & $2013 / 14$ & $2014 / 15$ \\
\hline & & \multicolumn{6}{|c|}{ Verbleibwahrscheinlichkeit bis Start Jg. 7} \\
\hline \multirow{3}{*}{$\begin{array}{l}\text { freie } \\
\text { Träger- } \\
\text { schaft }\end{array}$} & uneingeschränkt & $98,4 \%$ & $98,5 \%$ & $98,5 \%$ & $98,5 \%$ & $97,5 \%$ & $98,9 \%$ \\
\hline & eingeschränkt & $92,3 \%$ & $83,3 \%$ & $84,2 \%$ & $93,8 \%$ & $73,3 \%$ & $92,1 \%$ \\
\hline & ohne & - & $100,0 \% 5$ & $100,0 \%^{5}$ & $90,0 \%$ & $91,3 \%$ & $93,7 \%$ \\
\hline \multirow{3}{*}{$\begin{array}{l}\text { Wett- } \\
\text { bewerb } \\
\text { offen }\end{array}$} & uneingeschränkt & $97,8 \%$ & $96,7 \%$ & $97,3 \%$ & $93,5 \%$ & $95,3 \%$ & $96,1 \%$ \\
\hline & eingeschränkt & $91,2 \%$ & $88,2 \%$ & $81,4 \%$ & $92,9 \%$ & $81,7 \%$ & $88,2 \%$ \\
\hline & ohne & $100,0 \% 5$ & $100,0 \% 5$ & $77,4 \%$ & $60,0 \%$ & $45,8 \%$ & $82,0 \%$ \\
\hline
\end{tabular}

Quelle: eigene Berechnungen auf Datengrundlage eingereichter individueller Abgangsstatistiken der Schulen und von IT.NRW ${ }^{5}$

Umso erstaunlicher sind diese hohen Quoten, wenn man sie in andere Befunde zur Verbleibwahrscheinlichkeit von Schüler*innen ohne Gymnasialempfehlung am Gymnasium einordnet. So konnte in der Hamburger KESS-Studie aufgezeigt werden, dass mit dem Übertritt in die Jahrgangsstufe 7 noch 70,5 Prozent der Schüler*innen, die nach der Grundschule nicht über eine Gymnasialempfehlung verfügten, darin verblieben (Bos, Bonsen \& Gröhlich, 2009, S. 77). Insbesondere die Quote der NichtEmpfohlenen der beiden Gymnasien in freier Trägerschaft sticht dabei mit konstant über 90 Prozent verbliebener Schüler*innen heraus.

\section{Ausblick}

Im Resümee zeigt sich, dass Durchlässigkeit und Verbleibwahrscheinlichkeit in erster Linie in Abhängigkeit zu systembedingten, regionalen und schulspezifischen Kontextfaktoren stehen. Die Analyse von Daten aus der amtlichen Schulstatistik kann einen wichtigen Beitrag dazu leisten, dass sowohl auf administrativer Ebene in der überregionalen und kommunalen Schulverwaltung als auch im Profilbildungsprozess an den Einzelschulen Entwicklungen vorangetrieben werden, die auf passgenauere Konzepte im Zusammenspiel der beteiligten Akteure abzielen. Hierbei kann neben der Bereitstellung einzelschulbezogener Daten vor allem auch eine Individualstatistik auf Schüler*innenebene zur Verbesserung der Datenqualität beitragen. Individualdaten werden in NRW - im Gegensatz zu anderen Bundesländern - noch nicht erfasst und können von daher auch nicht für Detailanalysen bereitgestellt und

5 In diesen Jahrgängen wurden alle Schüler*innen ohne Gymnasialempfehlung in die siebte Jahrgangsstufe versetzt. Deren Anzahl war mit eins bis vier allerdings sehr gering. 
genutzt werden. Bei den einzelschulischen Selektionsquoten in diesem Beitrag handelt es sich um Individualdaten, die von den Schulen selbst zur Verfügung gestellt wurden. Vor diesem Hintergrund wäre eine Entwicklung hin zu schulstatistischen Individualdaten sehr zu begrüßen.

Darüber hinaus kann die Analyse solcher schüler*innenbezogener Daten genutzt werden, die einerseits mit den Funktionen von Schule und andererseits mit dem Bildungsauftrag des Gymnasiums verbunden sind. Daran schließen sich vor allem Fragen der Verteilungs- und Teilhabegerechtigkeit, aber auch der Anerkennungsgerechtigkeit an. Vor diesem Hintergrund wirft die von der fortschreitenden Öffnung forcierte Weiterentwicklung des Gymnasiums, seiner institutionellen Rahmenbedingungen und seines Bildungsverständnisses weitere Fragen auf: Was passiert im Quasi-Markt der kommunalen Schullandschaft, wenn sich Parameter des Wettbewerbs und der Profilierung ändern? Wie reagieren beispielsweise die zu einer Hochschulzugangsberechtigung führenden Schulformen auf bildungspolitische Entscheidungen wie die der flächendeckenden Wiedereinführung von G9, nicht nur in NRW? Tragen veränderte systemische Kontextfaktoren zu einer Stratifikation von Schulen derselben Schulform bei, wie dies am Beispiel der Solitär- und Sammelschulen gezeigt werden konnte? Entwickeln sich daraus schulspezifische Milieus?

Nicht zuletzt stellt sich die Frage, wie die Gymnasien ihrer durch die Öffnung erweiterten Schüler^innenklientel begegnen und wie sich darüber ihr Bildungsverständnis entwickelt. Welche schulkulturellen Maßnahmen ergreifen sie und welche pädagogischen Lösungen finden die Schulen angesichts der zunehmenden Heterogenität innerhalb ihrer gymnasialen Schülerschaft? Der Beitrag verdeutlicht, dass für die Gymnasien dabei Selektion nur eine eingeschränkte Option darstellt. Die starke Haltekraft von zuvor nicht oder nur eingeschränkt als gymnasial eingeschätzten Schüler*innen offenbart, dass diese Gymnasien eine Bereitschaft zur Anpassung an ihre Schülerschaft mitbringen und darüber hinaus Strategien entwickelt haben müssen, mit der neuen Schüler*innenklientel erfolgreich zu arbeiten.

\section{Literatur und Internetquellen}

Altrichter, H., Heinrich, M., \& Soukup-Altrichter, K. (2011a). Schulprofilierung: Annäherungen an ein Phänomen. In H. Altrichter, M. Heinrich \& K. Soukup-Altrichter (Hrsg.), Schulentwicklung durch Schulprofilierung? Zur Veränderung der Koordinationsmechanismen im Schulsystem (S. 11-45). Wiesbaden: Springer VS. https://doi. org/10.1007/ 978-3-531-92825-8_1

Altrichter, H., Heinrich, M., \& Soukup-Altrichter, K. (2011b). Governance-Regime der Schulentwicklung. In H. Altrichter, M. Heinrich \& K. Soukup-Altrichter (Hrsg.), Schulentwicklung durch Schulprofilierung? Zur Veränderung der Koordinationsmechanismen im Schulsystem (S. 217-239). Wiesbaden: Springer VS. https://doi.org/10.1007/978-3531-92825-8_7 
Baumert, J., Cortina, K. S., \& Leschinsky, A. (2003). Grundlegende Entwicklungen und Strukturprobleme im allgemeinbildenden Schulwesen. In K. S. Cortina, J. Baumert, A. Leschinsky, K. U. Mayer \& L. Trommer (Hrsg.), Das Bildungswesen in der Bundesrepublik Deutschland: Strukturen und Entwicklungen im Überblick (S. 52-147). Reinbek b. Hamburg: Rowohlt.

Bellenberg, G. (2012). Schulformwechsel in Deutschland - Durchlässigkeit und Selektion in den 16 Schulsystemen der Bundesländer innerhalb der Sekundarstufe I. Gütersloh: Bertelsmann.

Bos, W., Bonsen, M., \& Gröhlich, C. (Hrsg.). (2009). KESS 7 - Kompetenzen und Einstellungen von Schülerinnen und Schülern an Hamburger Schulen zu Beginn der Jahrgangsstufe 7. Münster: Waxmann.

Clausen, M., Winkler, C., \& Neu-Clausen, M. (2007). Die Förderung psychosozialer Entwicklung durch Schulprofile? Eine Analyse der Kontingentstundentafeln von Gymnasien in Baden-Württemberg. Zeitschrift für Pädagogik, 53 (6), 744-757.

DESTATIS (2018). Statistisches Jahrbuch 1976 bis 2015. Zugriff am 23.03.2019. Verfügbar unter: https://www.destatis.de/DE/Publikationen/StatistischesJahrbuch/StatistischesJahr buch_AeltereAusgaben.html.

Geißler, R. (2014). Bildungsexpansion und Wandel der Bildungschancen. Veränderungen im Zusammenhang von Bildungssystem und Sozialstruktur. In R. Geißler (Hrsg.), Die Sozialstruktur Deutschlands (S. 333-372). Wiesbaden: Springer VS. https://doi. org/10.1007/978-3-531-19151-5_13

Hermstein, B. (2018). Institutionelle Aktivierung im Schulsystem? Überlegungen am Beispiel lokaler Schulsystementwicklung unter Bedingungen von Regionalisierung. In T. Höhne, B. Hermstein \& S. Hartong (Hrsg.), Ökonomisierung von Schule? Bildungsreformen in nationaler und internationaler Perspektive (S. 130-153). Weinheim \& Basel: Beltz.

IT.NRW (2017). Einzelschulische Daten im Rahmen des Schulversuchs ,Abitur nach 12 oder 13 Jahren. Erhalten am 21.03.2017. Düsseldorf: Information und Technik NordrheinWestfalen.

Kussau, J., \& Brüsemeister, T. (2007). Governance, Schule und Politik - Zwischen Antagonismus und Kooperation. Wiesbaden: Springer VS.

Maroy, C., \& van Zanten, A. (2009). Regulation and Competition among Schools in Six European Localities. Sociologie du travail, (51), 67-79. https://doi.org/10.1016/j.soctra.2009.01.005

Mauthe, A., \& Rösner, E. (1998). Schulstruktur und Durchlässigkeit. In H.-G. Rolff, K.-O. Bauer, K. Klemm \& H. Pfeiffer (Hrsg.), Jahrbuch der Schulentwicklung, Bd. 10 (S. 87125). Weinheim: Juventa.

MSB NRW (Ministerium für Schule und Bildung des Landes Nordrhein-Westfalen) (2018). Das Schulwesen in Nordrhein-Westfalen aus quantitativer Sicht 2017/2018. Zugriff am 23.03.2019. Verfügbar unter: http://www.schulministerium.nrw.de/docs/bp/ Ministerium/Service/Schulstatistik/Amtliche-Schuldaten/Quantita_2016.pdf.

Rösner, E. (2013). Demografischer Wandel und seine Auswirkungen auf das Schulsystem. In G. Bellenberg \& M. Forell (Hrsg.), Bildungsübergänge gestalten. Ein Dialog zwischen Wissenschaft und Praxis (S. 181-188). Münster et al.: Waxmann.

Rösner, E., \& Stubbe, T. C. (2008). Übergangsentscheidungen und Schulerfolg im Zeichen demografischer Veränderungen. Ein Beitrag zur Gerechtigkeitsdebatte, zur Schulstruktur und zur Schulentwicklung. In W. Bos, H. G. Holtappels, H. Pfeiffer, H.-G. Rolff \& R. Schulz-Zander (Hrsg.), Jahrbuch der Schulentwicklung, Bd. 15 (S. 297-319). Weinheim: Juventa.

Schweizer, S. (2012). Die kanadische Bildungspolitik im Spiegel der drei Dimensionen des Föderalismus. In R. Hrbek, M. Große Hüttmann \& J. Schmid (Hrsg.), Bildungspolitik 
in Föderalstaaten und der Europäischen Union: Does Federalism Matter? (S. 164-179). Baden-Baden: Nomos. https://doi.org/10.5771/9783845241692-164

Tillmann, K.-J. (2008). Viel Selektion - wenig Leistung. Der PISA-Blick auf Erfolg und Scheitern in deutschen Schulen. In K. Böllert (Hrsg.), Von der Delegation zur Kooperation. Bildung in Familie, Schule, Kinder- und Jugendhilfe (S. 47-66). Wiesbaden: Springer VS. https://doi.org/10.1007/978-3-531-90846-5_3

Weiß, M. (2001). Quasi-Märkte im Schulbereich. Eine ökonomische Analyse. Zeitschrift für Pädagogik, 43. Beiheft (S. 69-85). Weinheim \& Basel: Beltz.

Zymek, B. (2009). Wettbewerb zwischen Schulen als Programm und Wettbewerb als Struktur des Schulsystems. In U. Lange, S. Rahn, W. Seitter \& R. Körzel (Hrsg.), Steuerungsprobleme im Bildungswesen. Festschrift für Klaus Harney (S. 81-100). Wiesbaden: Springer VS. https://doi.org/10.1007/978-3-531-91922-5_5

Matthias Forell, geb. 1984, Wissenschaftlicher Mitarbeiter in der AG Schulforschung an der Ruhr-Universität Bochum.

E-Mail: matthias.forell@rub.de

Gabriele Bellenberg, Prof. Dr., geb. 1967, Universitätsprofessorin für Schulforschung und Schulpädagogik am Institut für Erziehungswissenschaft der Ruhr-Universität Bochum.

E-Mail: gabriele.bellenberg@rub.de

Grit im Brahm, Prof. Dr., geb. 1975, Universitätsprofessorin für Unterrichtsentwicklung und Empirische Bildungsforschung am Institut für Erziehungswissenschaft der Ruhr-Universität Bochum.

E-Mail: grit.imbrahm@rub.de

Anschrift: Ruhr-Universität Bochum, Institut für Erziehungswissenschaft, 44801 Bochum 